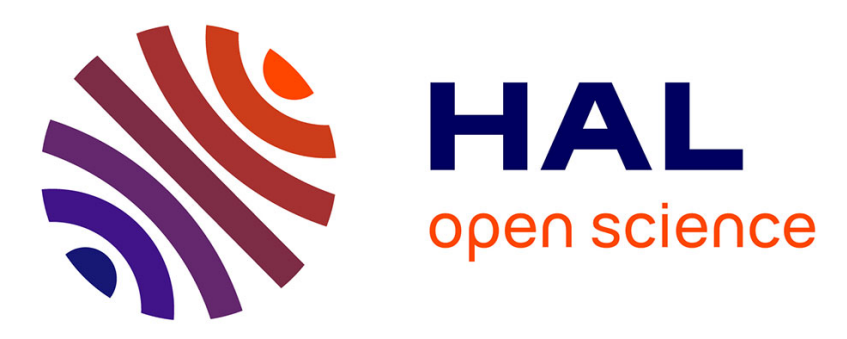

\title{
Les répercussions de la lombalgie chronique sont en partie liées à la perte du rôle social : une étude qualitative
}

Florian Bailly, Violaine Foltz, Sylvie Rozenberg, Bruno Fautrel, Laure Gossec

\section{- To cite this version:}

Florian Bailly, Violaine Foltz, Sylvie Rozenberg, Bruno Fautrel, Laure Gossec. Les répercussions de la lombalgie chronique sont en partie liées à la perte du rôle social : une étude qualitative. Revue du Rhumatisme, 2015, 82 (6), pp.396-401. 10.1016/j.rhum.2015.08.005 . hal-01256569

\section{HAL Id: hal-01256569 \\ https://hal.sorbonne-universite.fr/hal-01256569}

Submitted on 15 Jan 2016

HAL is a multi-disciplinary open access archive for the deposit and dissemination of scientific research documents, whether they are published or not. The documents may come from teaching and research institutions in France or abroad, or from public or private research centers.
L'archive ouverte pluridisciplinaire HAL, est destinée au dépôt et à la diffusion de documents scientifiques de niveau recherche, publiés ou non, émanant des établissements d'enseignement et de recherche français ou étrangers, des laboratoires publics ou privés. 


\section{Titre}

Les répercussions de la lombalgie chronique sont en partie liées à la perte du rôle social : une étude qualitative

\section{Auteurs :}

Florian Bailly ${ }^{1,2}$; Violaine Foltz ${ }^{1,2}$; Sylvie Rozenberg ${ }^{1,2}$; Bruno Fautrel $^{1,2}$; Laure Gossec $^{1,2}$

\section{AUTEUR CORRESPONDANT}

Dr. Laure Gossec, Hôpital Pitié-Salpétrière, Service de Rhumatologie, 47-83, boulevard de l'Hôpital - 75013 Paris France

laure.gossec@psl.aphp.fr

Tel: $+\underline{+33142178421}$

Fax: +33142177959

Affiliation de l'auteur correspondant : Hôpital Pitié-Salpétrière, Service de Rhumatologie, 47-83, boulevard de l'Hôpital - 75013 Paris France

\section{Affiliations}

1 AP-HP, Hôpital Pitié Salpêtrière, Service de Rhumatologie, Paris, F-75013, France ;

2-Sorbonne Universités, UPMC Univ Paris 06, Institut Pierre Louis d'Epidémiologie et de Santé Publique, F-75013, Paris, France 


\section{ABSTRACT}

Objectifs : La lombalgie chronique a un impact majeur sur la qualité de vie, lié à la douleur et à l'incapacité fonctionnelle mais également à cause de ses répercussions psychosociales. Le retentissement de la lombalgie chronique sur la participation sociale a été peu exploré. L'objectif de cette étude était de mieux comprendre le vécu des patients souffrant de lombalgie chronique en examinant plus particulièrement l'impact de cette maladie sur les relations familiales, sociales et professionnelles.

Méthodes : Etude qualitative monocentrique réalisée dans un centre hospitalier à Paris, France. Les participants étaient inclus s'ils souffraient de lombalgie commune chronique. Quatre entretiens collectifs semi-structurés (focus group) étaient organisés sur le thème du vécu lié à la lombalgie. Les verbatim collectés étaient classés, codifiés puis analysés selon la méthode d'analyse de contenu thématique.

Résultats: Vingt-cinq patients (11 hommes, 14 femmes) âgés de 25 à 81 ans participaient à cette étude. Une diminution de l'estime de soi était fréquemment observée, s'accompagnant d'un sentiment de honte et de frustration résultant des limitations fonctionnelles dans les activités de la vie quotidienne. L'impression prédominante chez les participants était d'être incompris et non soutenus, lié en partie à l'absence de symptômes visibles de cette maladie. Ils étaient victimes de la représentation collective négative de la lombalgie ("bénigne/maladie psychologique"). La lombalgie aboutissait chez certains patients à une perte significative de leur identité sociale, corrélée au sentiment d'impossibilité d'assurer leur rôle social dans leur environnement familial et professionnel. En revanche, la famille et les amis pouvaient avoir un rôle positif en aidant à gérer les douleurs.

Conclusion : Une évaluation systématique de l'impact social est conseillée dans la prise en charge des patients lombalgiques.

Mots clés : Lombalgie ; Recherche Qualitative ; Interaction Sociale 


\section{Introduction}

La lombalgie chronique est une pathologie fréquente qui a des répercussions importantes sur la qualité de vie [1] et engendre des coûts socio-économiques importants. Selon la classification de l'Organisation Mondiale de la Santé, cette pathologie s'inscrit dans une dimension bio-psycho-sociale au sein de laquelle la composante lésionnelle est en interaction avec d'autres facteurs [2]. Au delà de la douleur et de l'invalidité, les aspects psychosociaux pèsent de manière significative sur le vécu du patient lombalgique chronique [3-12] . La composante psychologique de la lombalgie chronique se manifeste par un état de détresse psychique tel que l'anxiété et la dépression [3-7]. Les patients développent différentes stratégies adaptatives (ou coping) - active ou passive, positive ou négative - pour faire face à la douleur et à son retentissement fonctionnel [8,9,11-13]. Les relations sociales peuvent également jouer un rôle important dans le vécu des patients lombalgiques $[4,14]$. Les contraintes psychosociales dans la vie personnelle (ex. faible soutien affectif) comme dans le milieu professionnel (faible soutien social au travail, insatisfaction professionnelle) constituent des facteurs de risque de chronicisation de la lombalgie [15]. "L'absence d'un interlocuteur attentif avec qui parler de la maladie" et "la mauvaise communication entre collègues de travail et avec la hiérarchie" sont considérés comme des "signes d'alerte jaune" identifiant des facteurs de risque de passage à la chronicité [16].

L'impact des relations familiales et sociales sur le vécu de la lombalgie chronique a été très peu exploré dans les études qualitatives [5,17-21] : Dans l'une d'entre elles, les participants se considéraient comme un fardeau pour leurs proches souffrant d'une incompréhension familiale croissante devant l'absence de diagnostic ou de symptomatologie franche [5]. Les autres études ne se sont pas intéressées de manière approfondie au rôle de la participation sociale dans le vécu de la maladie [17-21]. En revanche, les relations avec les professionnels de santé ont été fréquemment évaluées : assurer une prise en charge individualisée, informer et rassurer le patient, aborder les aspects psychosociaux et les options futures sont des mesures qui contribuent à améliorer les relations sociales [22-24]. II ressort des études qu'une vie sociale active favorise l'amélioration de l'état de santé physique 
[25]. Les aspects psychosociaux et relationnels sont indispensables non seulement pour mieux comprendre la lombalgie mais peuvent également orienter la prise en charge de cette pathologie [2]. L'approche multidisciplinaire de la lombalgie doit intégrer les aspects physiques de la maladie mais aussi, dans ce contexte, les interactions sociales ; celles-ci pouvant faire l'objet de programmes spécifiques de rééducation. Identifier le ressenti des patients souffrant de douleurs lombaires chroniques et la perception qu'ils ont de leurs relations aux autres, que ce soit dans la sphère privée ou au travail, pourrait permettre la mise en œuvre de mesures efficaces visant la réinsertion professionnelle des lombalgiques chroniques [2].

Les recherches ciblant les facteurs psychosociaux de la lombalgie chronique ont été principalement conduites à partir de questionnaires, de sondages ou de données épidémiologiques quantitatives. Les méthodes de recherche qualitative s'appuient sur le recueil, le classement et l'interprétation systématique des informations textuelles issues des entretiens ou des observations. Elles sont plus pertinentes que des études quantitatives pour aboutir à une meilleure compréhension du vécu des patients lombalgiques [26]. La présente étude avait pour objectif de mieux cerner le ressenti des patients lombalgiques chroniques en termes de participation sociale et de difficultés relationnelles et s'intéressait tout particulièrement aux répercussions de la maladie sur les relations familiales, sociales et professionnelles.

\section{Matériel et Méthodes}

Cette étude qualitative était basée sur la technique des focus group (entretiens collectifs) [27]. Les résultats ont été présentés selon les lignes directrices COREQ (Consolidated criteria for reporting qualitative research) [28]. La présente étude a été revue et approuvée par le comité d'éthique de la recherche de l'hôpital de la PitiéSalpêtrière après consentement écrit des participants.

\subsection{Recrutement et participants}

Les patients d'un Centre Hospitalier Universitaire et de deux cabinets de consultation parisiens ont été invités à participer à des entretiens collectifs (focus group) à 
condition de souffrir de lombalgie commune subaiguë ou chronique et de parler couramment le français ; au final, tous les patients inclus souffraient de lombalgie chronique. Une chirurgie récente du dos (moins de 3 mois) constituait l'unique critère d'exclusion. Une attention particulière a été portée à la sélection des participants en recherchant des caractéristiques démographiques variées (en particulier concernant l'âge et le niveau d'éducation). Les groupes étaient constitués d'un minimum de cinq participants pour garantir l'hétérogénéité des réponses et d'un maximum de dix participants pour faciliter les échanges au sein de chaque focus group.

\subsection{Caractéristiques des entretiens collectifs}

Pour guider la discussion de manière semi-directive, le modérateur disposait d'une grille d'entretien qui regroupait une série de questions d'ordre général et spécifiques. La technique des entretiens collectifs est particulièrement adaptée aux larges thématiques telles que "Vivre avec une lombalgie". Ce type d'étude qualitative a été préféré aux entretiens individuels afin de dynamiser les échanges, d'offrir un cadre de discussion plus agréable, de limiter l'anxiété des paticipants et de favoriser l'interaction de groupe. Chaque entretien, d'une durée de 90 à $120 \mathrm{mn}$, était animé par un rhumatologue - modérateur (LG) pendant qu'un second médecin observateur (FB) prenait des notes supplémentaires utilisées au moment de l'analyse des données. Avant le début de l'entretien, les caractéristiques démographiques des participants étaient collectées. Le modérateur posait ses questions à l'ensemble du groupe tout en encourageant l'implication individuelle et l'interaction des participants entre eux. Ces derniers étaient invités à décrire leur comportement, leurs croyances et leur état d'esprit vis à vis de la lombalgie et de ses répercussions. En premier lieu, l'impact général de la lombalgie était examiné, puis certains aspects plus spécifiques liés aux relations sociales (dans l'entourage familial, l'environnement social et professionnel) étaient abordés. La question générale introductive concernant les relations sociales était "Comment gérez-vous vos relations avec les autres face à votre lombalgie ?". Les relations avec les professionnels de la santé et les traitements de la lombalgie (médicaments, injections rachidiennes, école du dos ...) n'ont pas fait partie des thèmes abordés. En milieu de séance, le modérateur résumait les principaux sujets traités et les opinions des participants puis invitait ces derniers à développer d'autres aspects ou d'autres points de vue concernant la lombalgie. 


\subsection{Nombre de sujets}

Le principe directeur permettant de déterminer la taille adéquate d'un échantillon en recherche qualitative est la saturation des données, qui est atteinte lorsque la collecte n'apporte aucun élément nouveau ni aucune information supplémentaire par rapport aux données déjà collectées [29]. Ceci était le cas dans la présente étude.

\subsection{Analyse des données}

Les entretiens ont été enregistrés puis intégralement retranscrits. Deux chercheurs (FB et LG) étaient chargés d'analyser les transcriptions selon la méthode d'analyse de contenu thématique [30]. Quatre étapes ont été suivies : (I) Lecture itérative des entretiens pour obtenir un résumé contextualisé de la discussion puis choix des thèmes préliminaires. (II) Les verbatim des participants ont été identifiés puis codés. (III) Ces codes ont ensuite été regroupés dans des domaines thématiques. (IV) Synthèse des domaines pour établir des concepts. (V) Les domaines et concepts étaient alors soumis aux autres co-auteurs, spécialistes de la lombalgie. Par ailleurs, un des patients participant a été invité à valider les concepts obtenus. L'analyse des données étant réalisée de manière anonyme, il n'était pas possible d'établir un lien entre un commentaire et un participant spécifique. Les données qualitatives sont présentées sous forme de synthèses descriptives et illustrées par des commentaires tirés des transcriptions.

\section{Résultats}

\subsection{Participants}

L'échantillon était composé de 25 participants, 11 hommes et 14 femmes (Tableau 1). Les patients ont participé à l'un des 4 entretiens collectifs, chacun des entretiens étant composé de 5 à 9 participants. L'âge des participants variait de 25 à 81 ans (moyenne 50 ans). La durée de la maladie allait de 1 à 35 ans, avec une durée médiane de 10 ans. L'intensité moyenne de la douleur sur l'échelle visuelle analogique était de 4 sur 10 (0-8). Dans quatre-vingt pour cent des cas, la lombalgie s'accompagnait de douleurs radiculaires (en présence de douleurs radiculaires, la douleur lombaire était prédominante), 5 participants (20\%) étaient en arrêt de travail 
tandis que 16 (64\%) poursuivaient leur activité professionnelle, 2 étaient à la retraite, 1 était sans emploi et 1 était invalide. Seize participants (64\%) avaient bénéficié d'un programme intensif de rééducation du dos [31].

\subsection{Impact de la lombalgie}

Le principal effet négatif de la lombalgie sur la participation sociale est résumé à la figure 1.

\subsubsection{Image de soi dans un contexte de participation sociale}

La majorité des participants avaient une perception négative d'eux-mêmes, dans le cadre familial et professionnel (Figure 1), causée par la diminution de leurs capacités fonctionnelles mais également par la manière dont ils percevaient les réactions des autres : "Au travail, je me sens comme un poids mort, mon employeur préfère s'adresser à quelqu'un d'autre alors j'ai l'impression d'ennuyer tout le monde, je me sens inutile." Cette perception négative entraînait chez certains participants un sentiment de honte : "J'ai honte d'être handicapé, j'ai honte de demander l'aide de ma femme."

\subsubsection{Image renvoyée par autrui dans un contexte de participation sociale}

\section{Sentiment d'être incompris}

Du fait de la discordance entre la capacité physique diminuée et l'aspect physique normal d'un lombalgique, de nombreux patients se sentaient incompris. On observait souvent un déni de la maladie, comme si ces patients étaient atteints d'une pathologie imaginaire ou d'une maladie d'ordre purement psychologique ("Cette maladie ne se voit pas donc elle n'existe pas"). D'après un participant, seuls les bénéficiaires d'une intervention chirurgicale sont considérés comme gravement malades. "Les autres ont du mal à comprendre ma maladie : selon eux, l'absence de chirurgie signifie que je ne souhaite pas guérir". Certains participants avaient l'impression d'être perçus comme des paresseux et/ou des tricheurs "Au fond d'eux, 
les autres pensent que je suis un imposteur. Lorsque j'avoue souffrir de douleurs au dos, on me répond "ce n'est pas possible, tu es trop jeune pour avoir ce genre de problèmes".

\section{$\underline{\text { Le soutien de la famille et des amis }}$}

Dans certains cas, la famille et les amis avaient un rôle positif et bénéfique en aidant, en écoutant et en comprenant les difficultés du malade liées à la prise en charge de la douleur ; ils ont également motivé le patient à intensifier ses activités. "J'essaye toujours d'être en contact avec des associations plutôt que de rester seul. Le weekend, je tente d'organiser des activités avec mes enfants malgré mes douleurs lombaires. Je dois rester actif et ne pas hésiter à sortir car la vie continue." Avoir une vie sociale était souvent perçu comme un moyen efficace de porter son attention sur autre chose et d'oublier la douleur. Cela faisait partie de la prise en charge de la lombalgie. "Lorsque vous vous promenez ou vous installez à la terrasse d'un café pour boire un verre et que observez les gens, vous pensez à autre chose qu'à votre lombalgie. Aller au théâtre ou pratiquer d'autres loisirs permet d'oublier la maladie. II est possible de faire abstraction de la douleur même si celle ci est toujours présente, cela importe peu."

\section{Le rôle social dans l'environnement professionnel}

Le rôle social était profondément modifié au travail en raison des difficultés à maintenir une productivité constante et de la grande variabilité du degré d'incapacité fonctionnelle causée par la lombalgie. A titre d'exemple, un participant rapportait que quelques jours après son retour au travail, son employeur avait déjà oublié qu'il souffrait de problèmes de dos. Ce dernier avait réalisé "quelques aménagements mineurs de son poste de travail tels que la mise en place d'un siège ergonomique, pour éviter [d'autres mesures]' sans considérer la lombalgie comme une maladie chronique. "Il arrive parfois que la douleur m'empêche de quitter mon lit alors que j'ai le désir de me lever. Amener mon patron à comprendre tout cela s'avère mission impossible. La planification du travail ne prend pas en compte notre maladie et nous sommes obligés de nous y conformer." Le sentiment de ne pas pouvoir assumer son 
rôle social au travail et d'avoir à compter sur l'aide de ses collègues était présent chez plusieurs participants. "Je ne me sens pas à ma place, je délègue beaucoup de travail à mes collègues et me décharge sur eux. Comme j'ai besoin d'aide en permanence, ils font des heures supplémentaires pour compenser la surcharge de travail."

\subsubsection{Le rôle social et les croyances sociétales en lien avec la lombalgie}

\section{$\underline{\text { Une maladie fréquente }}$}

L'incompréhension dont se sentaient victimes les patients s'expliquait sans doute par la forte prévalence de la lombalgie. Appelée "la maladie du siècle", cette pathologie fréquente n'était toutefois pas perçue comme une maladie grave et débilitante. "Lorsque que je dis à mon entourage que je souffre de douleurs lombaires, on me répond : ce n'est rien, c'est transitoire, ça va s'arranger. C'est courant d'avoir mal au dos, tu n'as pas un cancer".

\section{$\underline{\text { Une maladie bénigne }}$}

Deux participantes ont établi une comparaison avec des maladies considérées comme "graves" : un cancer du sein pour l'une et une sclérose en plaque pour l'autre. Selon leur vécu, le retentissement social était plus important en cas de lombalgie que dans le cas de ces maladies graves.

\section{$\underline{\text { Image psychologique négative de la lombalgie }}$}

La lombalgie était considérée comme une maladie psychologique car sans symptomatologie physique visible susceptible d'expliquer la douleur. L'entourage recommandait au malade de consulter un psychologue pour trouver une solution à ses douleurs. "On me demande parfois : "est-ce que vous avez mal ?" mais également "avez-vous des problèmes personnels? II serait peut être bon de voir un psychiatre ..." comme si ma maladie était une invention, comme si le problème était dans ma tête et non dans mon dos." Ces remarquent mettent en évidence la stigmatisation de la lombalgie et du patient lombalgique. 


\subsubsection{Perte significative de la fonction sociale}

Les participants avaient l'impression d'être incapables d'assumer leur rôle social dans le cadre familial mais aussi professionnel. Certains d'entre eux déclaraient ne plus pouvoir porter leur enfant ni même s'en occuper. "Père de deux jeunes enfants, je ne peux pas les emmener se promener, je ne peux pas les porter". Les hommes avaient parfois le sentiment de perdre leur masculinité en raison de leur incapacité à porter des objets lourds tels que des bagages. N'ayant pu aider des amis à effectuer une tâche exigeant un effort physique, l'un d'entre eux confiait ressentir une grande frustration, surtout compte tenu de sa robustesse physique (il était grand et athlétique). "Par exemple, lorsque nous partons en vacances, c'est ma femme qui porte les bagages. Tout le monde me regarde, et comme je suis très musclé les gens ne comprennent pas pourquoi ce n'est pas moi qui m'en charge. Dans le métro, les femmes me demandent parfois de les aider à soulever la poussette de leur enfant et je suis contraint de leur répondre que je ne peux pas les aider. Je suis jugé comme un goujat." Deux hommes ont également évoqué brièvement la perte de leur virilité dans les relations intimes mais ce thème n'a pas fait l'objet d'une analyse approfondie dans le focus group.

\subsection{Anxiété, dépression et stratégie d'adaptation (coping) étaient des composantes majeures de la lombalgie}

La plupart des participants souffraient d'anxiété associée à la peur de l'avenir en raison de l'évolution imprévisible de la maladie et des rechutes inattendues. Parmi eux, certains présentaient également des signes de dépression. Les stratégies d'adaptation à la douleur ont été décrites comme un long processus. Une modification de ces conduites d'adaptation pendant la durée de la maladie était rapportée chez certains participants. Ces aspects ont été retrouvés dans d'autres études [3-7] mais n'ont pas fait l'objet d'une analyse approfondie dans la présente étude.

\section{Discussion}


Cette étude a mis en évidence le retentissement négatif majeur de la lombalgie chronique sur la perception de soi ; sur l'incompréhension dont fait preuve l'entourage et les difficultés de socialisation dans l'environnement familial et professionnel. Ces difficultés conduisaient les patients lombalgiques à ressentir une perte significative de leur identité sociale. Par ailleurs, favoriser le maintien des relations sociales pourrait s'avérer bénéfique aux patients lombalgiques, une approche sociale positive devrait être encouragée pour réduire l'impact de la maladie.

Toutefois, cette étude comportait des limites. II s'agissait d'une étude monocentrique incluant 25 patients ; cependant le recrutement dans un centre de soins tertiaires et en consultation de ville mais aussi la sélection de profils démographiques très variés ont conduit à une grande diversité des caractéristiques des participants. De plus, le nombre de participants n'a pas été fixé arbitrairement, les entretiens collectifs ayant été poursuivis jusqu'à saturation des données. Le nombre d'entretiens et de participants correspondait à celui observé dans d'autres études utilisant la méthode des focus group [29]. Cette étude a été menée par des rhumatologues tandis que la majorité des études dans ce domaine sont conduites par des psychologues : dans une récente revue des études qualitatives sur la lombalgie [4], une seule étude [18] sur 33 [5-10,13,19-23,32] était menée par des médecins. C'est pourquoi, dans le cas présent, certaines composantes du vécu de la lombalgie ont pu être sousestimées. Cet aspect de l'étude constitue cependant une force : en effet, il autorisait une approche plus clinique en raison de l'expérience des rhumatologues dans le domaine de la lombalgie et permettait probablement d'analyser de manière plus ciblée les données. La vie intime, y compris les difficultés sexuelles, n'a pas été explorée étant donné la configuration collective des entretiens. Cependant, les entretiens collectifs apparaissent comme une technique pertinente pour le recueil des données qualitatives et produisent, d'après certains auteurs, des informations bien plus complètes que les entretiens individuels [33].

La plupart des participants exerçaient leur activité professionnelle sans être en arrêt de travail et présentaient un score douleur moyen sur l'échelle visuelle analogique de 4/10. Ces résultats sont sans doute le signe d'une stratégie adaptative (coping) 
appropriée des participants ou s'expliquent par la présence d'une lombalgie sans troubles majeurs. Ils peuvent être secondaires à la durée prolongée de la maladie : la majorité des patients révélaient avoir eu recours à plusieurs arrêts de travail ou un congé maladie longue durée mais avaient repris leur activité professionnelle au moment de l'étude. Par ailleurs, les deux tiers des patients avaient bénéficié de soins spécialisés dans un centre de soins tertiaires dont l'objectif était d'encourager le retour à la vie professionnelle.

Le rôle de la famille et des amis était perçu différemment en fonction des participants. Certains d'entre eux reconnaissaient les bienfaits considérables de l'attitude compréhensive et bienveillante de leur entourage tandis que d'autres déploraient l'absence de soutien familial et social. Au-delà d'une écoute et d'une compréhension actives, le soutien de l'entourage pourrait favoriser la motivation et la volonté des lombalgiques chroniques à intensifier leurs activités [34]. Au cours de différentes études menées sur des patients souffrant de douleurs chroniques du dos, incluant des enfants [35], l'attitude du système familial et des pairs conditionnait l'expérience du patient douloureux chronique. Pour améliorer le traitement des douleurs chroniques chez l'enfant et l'adolescent, un programme pluridisciplinaire intensif était mis en œuvre, invitant les parents à participer à la prise en charge et s'appuyant sur la réponse des parents et les interactions positives face à la douleur [35]. C'est pourquoi, il semblerait intéressant d'intégrer au programme de prise en charge de la lombalgie des séances de formation destinées aux proches des malades.

Les différentes attitudes face à la lombalgie sont conditionnées par la représentation sociale négative de cette maladie souvent considérée comme à composante purement "psychologique". La stigmatisation des patients lombalgiques a été fréquemment évoquée dans les études qualitatives [3]. L'absence de lésions physiquement visibles contribue probablement à renforcer cette stigmatisation. De plus, la lombalgie affecte principalement la tranche d'âge des 20-50 ans qui peut conduire les personnes extérieures à ne considérer que la force et santé physique du patient, source d'incompréhension et de stigmatisation. Le rôle des médias qui dépeignent les lombalgiques chroniques comme des personnes aux intentions frauduleuses, motivées par les compensations financières a également été évoqué. Un programme informatif et éducatif sur la lombalgie devrait être mis en place à 
l'intention non seulement des patients et de leur entourage (comme mentionné plus haut) mais surtout du grand public. La diffusion de livrets d'information tels que le "Back book" ou "Guide du dos" [36] pourrait ainsi améliorer la perception collective de cette maladie et encourager le soutien social des malades, dans le but de faciliter la réinsertion des patients lombalgiques dans leur environnement personnel.

Les répercussions de la lombalgie sur l'activité professionnelle sont bien connues et associées à un risque d'arrêt de travail prolongé [37]. Une meilleure connaissance des facteurs relationnels en milieu professionnel est primordiale pour traiter et prévenir l'incapacité au travail liée à la lombalgie. Une étude récente [38] a souligné que pour la plupart des patients nécessitant une prise en charge médicale, la diminution de la douleur n'est pas le seul résultat ou objectif de traitement à prendre en compte. La participation sociale au travail et la restauration fonctionnelle jouent un rôle majeur dans la récupération du patient et sont bénéfiques à l'état de santé général et au bien être mental. Dans la présente étude, les participants aux entretiens collectifs ont souligné la nécessité de prendre en compte la perte du rôle social pour garantir une réinsertion professionnelle progressive.

La lombalgie n'est pas la seule pathologie qui mène à une altération de la fonction sociale. Un tel retentissement se retrouve également dans d'autres pathologies chroniques telles que l'arthrose, la polyarthrite rhumatoïde, le diabète de type 2 , la spondylarthrite ou l'AVC. La plupart des maladies chroniques ont un impact psychologique comme la dépression, en partie lié à la perte du rôle social. La rééducation des patients victimes d'un AVC a démontré que l'amélioration de l'état dépressif était conditionnée par le fonctionnement social du patient [39]. Cette corrélation pourrait s'appliquer aux patients lombalgiques et constitue un autre argument en faveur de mesures visant à améliorer le rôle social du patient. Cependant, dans la présente étude, la dépression proprement dite n'était pas évaluée.

Cette étude analysait les composantes sociales de la lombalgie chronique ; une prise en charge holistique de la lombalgie devrait tenir compte des interactions sociales et aiderait à mieux identifier les difficultés rencontrées et les améliorations requises. La composante sociale du modèle biopsychosocial n'est pas suffisamment représentée dans les évaluations des patients. D'autres études seront nécessaires pour permettre 
l'élaboration d'un questionnaire centré sur la composante sociale : ce questionnaire sera ainsi l'occasion pour les patients et les soignants de détecter les domaines à améliorer, la finalité ultime étant d'améliorer les résultats de la prise en charge de la lombalgie chronique. 


\section{Financement :}

Cette étude a été rendue possible grâce à une subvention de la Société Française de Rhumatologie.

Conflits d'intérêts : Aucun

Remerciements : Nous remercions l'ensemble des patients qui ont accepté de participer à cette étude qualitative ainsi que le patient-partenaire qui a validé les résultats. 


\section{Bibliographie}

[1] Vos T, Flaxman AD, Naghavi M, Al. A. Years lived with disability (YLDs) for 1160 sequelae of 289 diseases and injuries 1990-2010: a systematic analysis for the Global Burden of Disease Study 2010. Lancet. 2012 Dec 15;380(9859):2163-96.

[2] Waddell G, Burton a K. Concepts of rehabilitation for the management of low back pain. Best Pract Res Clin Rheumatol. 2005 Aug;19(4):655-70.

[3] Bunzli S, Watkins R, Smith A et al. Lives on Hold: A Qualitative Synthesis Exploring the Experience of Chronic Low-back Pain. Clin J Pain. 2013 Jan 30;29(10):907-16.

[4] Snelgrove S, Liossi C. Living with chronic low back pain: a metasynthesis of qualitative research. Chronic IIIn. 2013 Apr 12;9(4):283-301.

[5] Vroman K, Warner R, Chamberlain K. Now let me tell you in my own words: narratives of acute and chronic low back pain. Disabil Rehabil. 2009 Jan;31(12):976-87.

[6] Snelgrove S, Edwards S, Liossi C. A longitudinal study of patients' experiences of chronic low back pain using interpretative phenomenological analysis: changes and consistencies. Psychol Health. 2013 Jan;28(2):121-38.

[7] Osborn M, Smith JA. Living with a body separate from the self. The experience of the body in chronic benign low back pain: an interpretative phenomenological analysis. Scand J Caring Sci. 2006 Jun;20(2):216-22.

[8] Busch H. Appraisal and coping processes among chronic low back pain patients. Scand J Caring Sci. 2005;19:396-402.

[9] Young AE, Wasiak R, Phillips $L$ et al. Workers' perspectives on low back pain recurrence: "it comes and goes and comes and goes, but it's always there". Pain. 2011;152:204-11.

[10] Raak R, Wahren LK. Health experiences and employment status in subjects with chronic back pain: a long-term perspective. Pain Manag Nurs Off J Am Soc Pain Manag Nurses. 2006;7:64-70.

[11] Keefe FJ, Crisson J, Urban BJ et al. Analyzing chronic low back pain: the relative contribution of pain coping strategies. Pain. 1990 Mar;40(3):293-301.

[12] Cano A. Pain catastrophizing and social support in married individuals with chronic pain: the moderating role of pain duration. Pain. 2004 Aug;110(3):65664.

[13] De Souza LH, Frank AO. Experiences of living with chronic back pain: the physical disabilities. Disabil Rehabil. 2007 Apr 15;29(7):587-96. 
[14] Glenton C. Chronic back pain sufferers--striving for the sick role. Soc Sci Med. 2003 Dec;57(11):2243-52.

[15] Hoogendoorn WE, van Poppel MN, Bongers PM et al. Systematic review of psychosocial factors at work and private life as risk factors for back pain. Spine (Phila Pa 1976). 2000 Aug 15;25(16):2114-25.

[16] Kendall NA, Linton SJ, Main CJ. Guide to Assessing Psychosocial Yellow Flags in Acute Low Back Pain: Risk Factors for Long-Term Disability and Work Loss. Wellington, New Zeal Accid Rehabil Compens Insur Corp New Zeal Nathional Heal Commitee. 1997;

[17] Cook FM, Hassenkamp A-M. Active Rehabilitation for Chronic Low Back Pain. Physiotherapy. 2000 Feb;86(2):61-8.

[18] Tavafian SS, Gregory D, Montazeri A. The experience of low back pain in Iranian women: a focus group study. Health Care Women Int. 2008 Apr;29(4):339-48.

[19] Campbell C, Guy A. "Why can”t they do anything for a simple back problem?' A qualitative examination of expectations for low back pain treatment and outcome. J Health Psychol. 2007 Jul;12(4):641-52.

[20] Corbett M, Foster NE, Ong BN. Living with low back pain-Stories of hope and despair. Soc Sci Med. 2007 Oct;65(8):1584-94.

[21] Smith JA, Osborn M. Pain as an assault on the self: An interpretative phenomenological analysis of the psychological impact of chronic benign low back pain. Psychol Health. Routledge; 2007 Jul;22(5):517-34.

[22] Slade SC, Molloy E, Keating JL. "Listen to me, tell me": a qualitative study of partnership in care for people with non-specific chronic low back pain. Clin Rehabil. 2009 Mar;23(3):270-80.

[23] Cooper K, Smith BH, Hancock E. Patients' perceptions of self-management of chronic low back pain: evidence for enhancing patient education and support. Physiotherapy. 2009;95:43-50.

[24] Laerum E, Indahl A, Skouen JS. What is "the good back-consultation"? A combined qualitative and quantitative study of chronic low back pain patients' interaction with and perceptions of consultations with specialists. J Rehabil Med Off J UEMS Eur Board Phys Rehabil Med. 2006;38:255-62.

[25] Takeyachi Y, Konno S, Otani K et al. Correlation of low back pain with functional status, general health perception, social participation, subjective happiness, and patient satisfaction. Spine (Phila Pa 1976). $2003 \mathrm{Jul}$ 1;28(13):1461-6; discussion 1467.

[26] Malterud K. Qualitative research: standards, challenges, and guidelines. Lancet. 2001 Aug 11;358(9280):483-8. 
[27] Britten N. Qualitative Research Qualitative interviews in medical research. Br Med J. 1995;311:251-3.

[28] Tong A, Sainsbury P, Craig J. Consolidated criteria for reporting qualitative research (COREQ): a 32-item checklist for interviews and focus groups. Int J Qual Heal .... 2007;19(6):349-57.

[29] Carlsen B, Glenton C. What about N? A methodological study of sample-size reporting in focus group studies. BMC Med Res Methodol. 2011;11:26.

[30] Crabtree B, Miller W. Doing Qualitative Research. SAGE; 1999.

[31] Poulain C, Kernéis S, Rozenberg S et al. Long-term return to work after a functional restoration program for chronic low-back pain patients: a prospective study. Eur Spine J. 2010;19:1153-61.

[32] Benjaminsson O, Biguet G, Arvidsson I et al. Recurrent low back pain: relapse from a patients perspective. J Rehabil Med Off J UEMS Eur Board Phys Rehabil Med. 2007;39:640-5.

[33] Krueger RA, Casey MA. Focus Groups; a practical guide for applied research. 3rd edition. Inc. SP, editor. 2000.

[34] Mathy C, Broonen J-P, Henrotin Y et al. Development and validation of a questionnaire assessing volitional competencies to enhance the performance of physical activities in chronic low back pain patients. BMC Musculoskelet Disord. 2011 Jan;12:111.

[35] Logan DE, Engle LB, Feinstein AB et al. Ecological system influences in the treatment of pediatric chronic pain. Pain Res Manag. 2012;17(6):407-11.

[36] Symonds TL, Burton AK, Tillotson KM et al. Absence resulting from low back trouble can be reduced by psychosocial intervention at the work place. Spine (Phila Pa 1976). 1995;20:2738-45.

[37] Steenstra IA, Verbeek JH, Heymans MW et al. Prognostic factors for duration of sick leave in patients sick listed with acute low back pain: a systematic review of the literature. Occup Environ Med. 2005 Dec;62(12):851-60.

[38] Costa-Black KM, Loisel P, Anema JRet al. Back pain and work. Best Pract Res Clin Rheumatol. 2010 Apr;24(2):227-40.

[39] Schmid AA, Damush T, Tu W, Bakas Tet al. Depression improvement is related to social role functioning after stroke. Arch Phys Med Rehabil. 2012 Jun;93(6):978-82.

[40] Froud R, Patterson S, Eldridge $S$ et al. A systematic review and meta-synthesis of the impact of low back pain on people's lives. BMC Musculoskelet Disord. 2014 Jan;15:50. 
Tableau 1. Caractéristiques des participants des focus group

\begin{tabular}{|l|l|l|l|l|l|}
\hline & $\begin{array}{l}\text { Echantillon } \\
\text { total, N=25 }\end{array}$ & $\begin{array}{l}\text { Focus } \\
\text { group 1, } \\
\mathrm{N}=7\end{array}$ & $\begin{array}{l}\text { Focus } \\
\text { group 2, } \\
\mathrm{N}=6\end{array}$ & $\begin{array}{l}\text { Focus } \\
\text { group 3, } \\
\mathrm{N}=7\end{array}$ & $\begin{array}{l}\text { Focus } \\
\text { group 4, } \\
\mathrm{N}=5\end{array}$ \\
\hline $\begin{array}{l}\text { Age, années, } \\
\text { moyenne (étendue) }\end{array}$ & $51(25-81)$ & $\begin{array}{l}49(42- \\
59)\end{array}$ & $\begin{array}{l}54,5(32- \\
81)\end{array}$ & $\begin{array}{l}46(29- \\
56)\end{array}$ & $\begin{array}{l}60(34- \\
66)\end{array}$ \\
\hline $\begin{array}{l}\text { Sexe, masculin, N } \\
(\%)\end{array}$ & $11(44 \%)$ & $3(43 \%)$ & $2(33 \%)$ & $5(71 \%)$ & $1(20 \%)$ \\
\hline $\begin{array}{l}\text { Durée de la } \\
\text { lombalgie, années, } \\
\text { moyenne (étendue) }\end{array}$ & $10(1-35)$ & $2(1-35)$ & $7(2-20)$ & $15(4-25)$ & $10(2-25)$ \\
\hline $\begin{array}{l}\text { Intensité de la } \\
\text { douleur EVA (0-10), } \\
\text { moyenne (étendue), }\end{array}$ & $4(0-8)$ & $4(0-7)$ & $4(0-8)$ & $3(0-4)$ & $6(0-7)$ \\
\hline
\end{tabular}

\section{Figures}

Figure 1 (dans un fichier séparé) : Synthèse du contenu thématique rapporté pendant les 4 entretiens collectifs au sujet des répercussions sociales de la lombalgie 


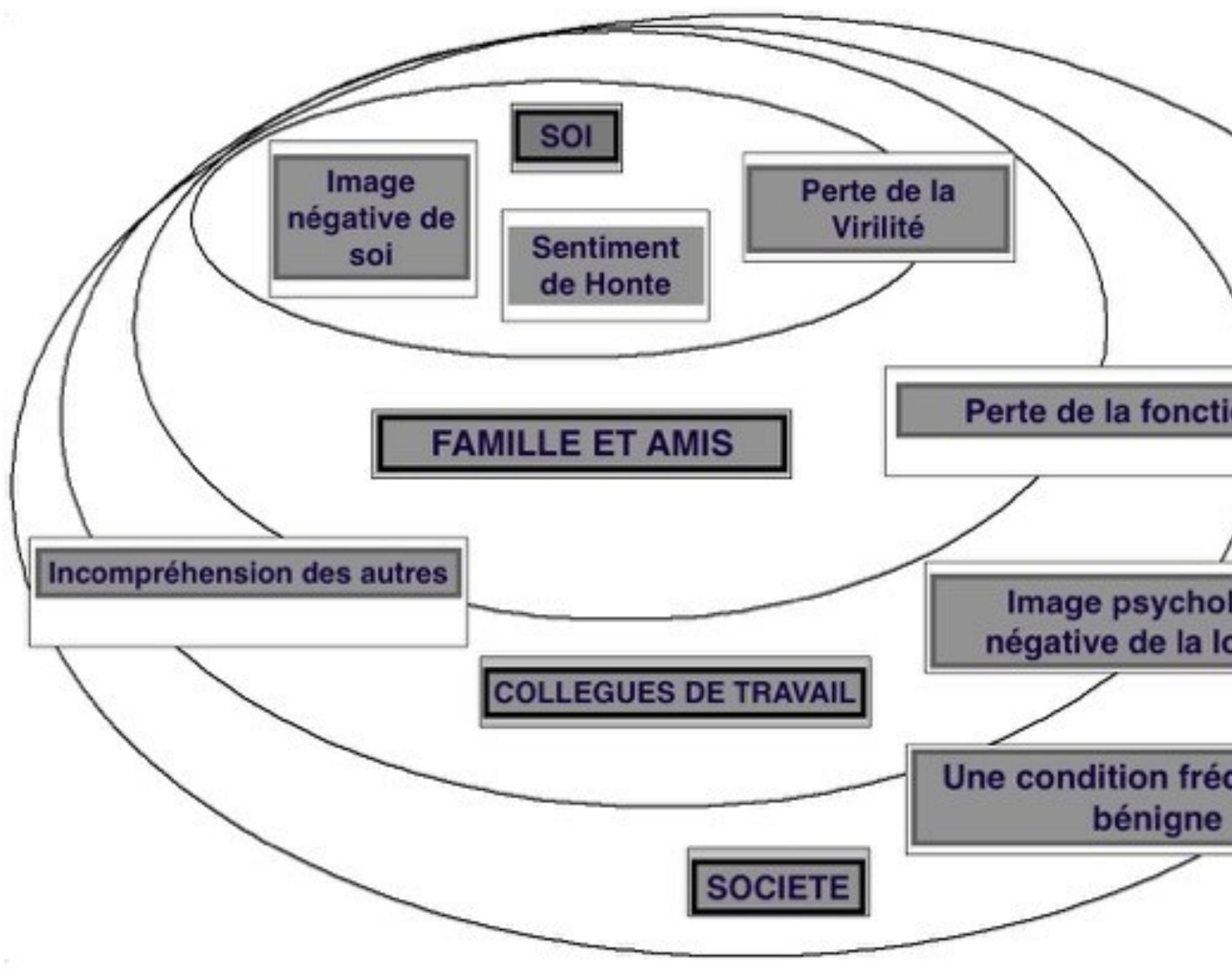

